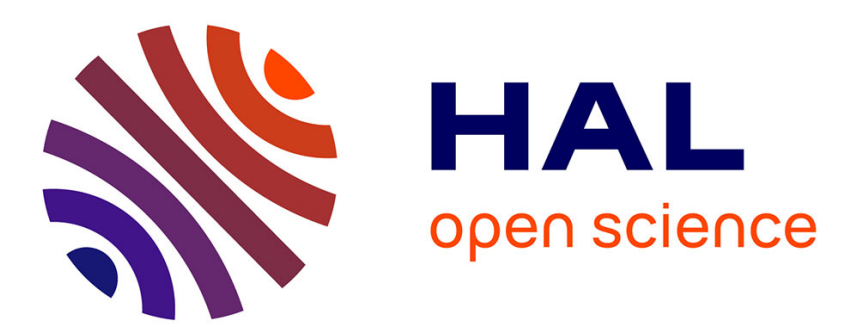

\title{
From telephone cords to branched buckles: A phase diagram
}

\author{
Jean-Yvon Faou, Sergey Grachev, Etienne Barthel, Guillaume Parry
}

\section{To cite this version:}

Jean-Yvon Faou, Sergey Grachev, Etienne Barthel, Guillaume Parry. From telephone cords to branched buckles: A phase diagram. Acta Materialia, 2017, 125, pp.524 - 531. 10.1016/j.actamat.2016.12.025 . hal-01447459

\section{HAL Id: hal-01447459 \\ https://hal.science/hal-01447459}

Submitted on 26 Jan 2017

HAL is a multi-disciplinary open access archive for the deposit and dissemination of scientific research documents, whether they are published or not. The documents may come from teaching and research institutions in France or abroad, or from public or private research centers.
L'archive ouverte pluridisciplinaire HAL, est destinée au dépôt et à la diffusion de documents scientifiques de niveau recherche, publiés ou non, émanant des établissements d'enseignement et de recherche français ou étrangers, des laboratoires publics ou privés. 


\title{
From telephone cords to branched buckles : a phase diagram
}

\author{
J.-Y. Faou ${ }^{\mathrm{a}}$, Sergey Grachev ${ }^{\mathrm{a}}$, Etienne Barthel ${ }^{\mathrm{a}}$, Guillaume Parry ${ }^{\mathrm{b}, \mathrm{c}, 1}$ \\ ${ }^{a}$ Surface du Verre et Interfaces, UMR 125 CNRS/Saint-Gobain, 39, quai Lucien Lefranc, \\ F-93303 Aubervilliers, Cedex, France. \\ ${ }^{b}$ Univ. Grenoble Alpes, SIMAP, F-38000 Grenoble, France \\ ${ }^{c}$ CNRS, SIMAP, F-38000 Grenoble, France
}

\begin{abstract}
Thin films with low adhesion and large residual stresses may buckle and delaminate from their substrates. This delamination often results in oscillating patterns known as 'telephone cords'. Other configurations can be observed as well, such as entangled networks of blisters. The present study aims at elucidating how these networks are generated through branching. A model coupling a geometrically non-linear plate model and a cohesive zone with mode dependent interfacial toughness is used. Previous work on the early stage delamination of initially circular blisters during interfacial crack front propagation are revisited and extended to the remote post-critical regime to capture front branching. We also build a partial phase diagram of buckling driven delamination morphologies. An intrinsic critical stress parameter is proposed which can be used to predict delamination morphology and to define 'safe' conditions where buckle propagation can be avoided. This critical stress also defines an intrinsic lengthscale which plays the role of a minimum nucleation size.
\end{abstract}

Keywords:

Buckling, Delamination, Finite element method, Cohesive zone model, Telephone cord, mode-mixity

\footnotetext{
${ }^{1}$ Corresponding author. Email address: guillaume.parry@simap.grenoble-inp.fr
} 


\section{List of notations}

$\begin{array}{ll}E, \nu & \text { Young's modulus and Poisson's ratio of the film } \\ h & \text { Film thickness } \\ \delta_{i j} & \text { Kronecker symbol } \\ \sigma_{i j}=-\sigma_{0} \delta_{i j} & \text { Equibiaxial stress tensor in the film prior to buckling }\left(\sigma_{0}>0\right) \\ E^{*}=E /(1-\nu) & \text { For an equibiaxial loading } \sigma_{0}=E^{*} \epsilon_{0} \\ \sigma_{c} & \text { Critical buckling stress } \\ \psi & \text { Mode mixity parameter } \\ G_{0} & \text { Strain energy per unit area stored in the film } \\ G_{c} & \text { Toughness of the film/substrate interface } \\ G_{I c} & \text { Pure mode I interface toughness } \\ \eta & \text { Parameter defining the interface sensitivity to mode mixity } \\ \bar{T} & \text { Interface traction vector } \\ \left(T_{n}, T_{t}\right) & \text { Normal and tangential components of } \bar{T} \\ \left(T_{n}^{0}, T_{t}^{0}\right) & \text { Maximum normal and tangential components allowed for } \bar{T} \\ \left(T_{n}^{i}, T_{t}^{i}\right) & \text { Normal and tangential components of } \bar{T} \text { actually reached at peak traction }\end{array}$

\section{Introduction - Phases of thin film blisters}

Thin film blisters form because as the coating buckles it breaks the film/substrate interface. The shape of the buckle is eventually controlled by the coupling between these two complex problems. Buckling has been intensively studied in the framework of the Föppl-Von Kàrmàn thin plate equilibrium equations [1, 2]. A coating with large compressive stresses can buckle above a critical stress $\sigma_{c}$. In thin films the internal stress incurred during deposition can easily exceed this critical buckling stress. The post-critical regime is characterized by large out-of-plane displacements, so the geometrically nonlinear character of the plate equations plays a significant role, and analytical solutions exist only in the simplest cases. The difficulty is even greater if, instead of fixed boundaries, the process of delamination itself is taken into account. The only analytical results available in the post-critical regime are the straight-sided blister (i.e. the Euler column) and the circular blister [2], characterized by their simple geometries. With the help of numerical calculations, some of the mysteries surrounding the ubiquitous 'telephone cord buckle' (TCB) [3-5] have been unraveled. Various studies [6-10] identified the TCB as resulting from the secondary buckling of a straight-sided blister. Recently, significant progress have been made in understanding the TCB propagation mechanism by coupling geometrical nonlinearity for the plate with a cohesive zone for the interface [11]. In this respect, the mode mixity dependence of interfacial toughness proved to be a key point. In particular, the model allowed for a very good understanding of the relationship between adhesion and blister morphology with a good correlation with experiments [12].

The morphology of a telephone cord blister on a rigid substrate can now be anticipated given full details about elastic properties, coating thickness, residual stresses and adhesion, including mode mixity dependence. However, the question of blister bifurcation (or "branching") remains open. Indeed, a large variety 
of blister configurations can be observed experimentally. For example, in Fig.1a, we show isolated telephone cords obtained with Mo thin films (see below). Decreasing film thickness and increasing residual stresses we observe entangled networks of telephone cords (Fig. 1-b) with separation of the delamination front into several branches at particular steps of the delamination process.
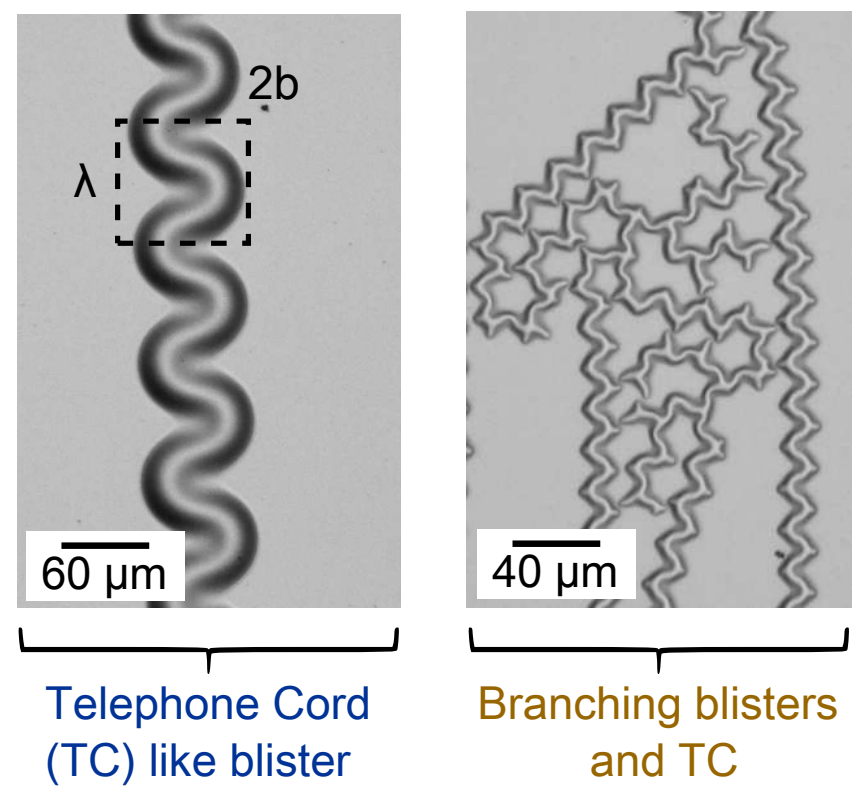

Figure 1: Buckles formed with molybdenum coatings deposited on a silicon wafer with a thin Ag interlayer. Two buckling delamination patterns observed experimentally: (a) isolated telephone cords blisters; (b) entangled networks of telephone cords (branching blisters) for reduced film thickness.

The aim of this paper is to determine conditions under which blisters branch. The case of the initial destabilization of a circular blister, originally studied in [13], is first investigated. It contains the key features for the analysis of the delamination front destabilization and branching and serves as a reference case for our numerical study. Then we build a partial phase diagram for blister morphologies which we also illustrate with experimental observations.

\section{Methods}

The numerical scheme and experimental methods are as described in our previous papers [11, 12].

In brief, the FEM model (Fig. 2) consists of plate elements with geometrical nonlinearity bonded to a rigid substrate. To generate compressive residual stresses in the simulation, we start from a relaxed film and apply a uniform thermal expansion to the film with components $\epsilon_{x x}=\epsilon_{y y}=\epsilon_{0}$ and $\epsilon_{x y}=0$. This expansion (the so called eigenstrain, which is here positive, with $\epsilon_{0}>0$ ) 
is in fact restrained by the rigid substrate, so that the actual total film strain is zero. At equilibrium, the eigenstrain is therefore compensated by an exactly opposite elastic contraction, which generates an equibiaxial compressive stress state $\sigma_{x x}=\sigma_{y y}=-E /(1-\nu) \epsilon_{0}=-\sigma_{0}, \sigma_{x y}=0$.

Interface debonding is described by mixed-mode cohesive elements inserted between the plate elements and the substrate. The traction vs. separation law is linear/softening. It is linear and reversible $(\bar{T}=K \bar{\delta}$ where $K$ is the stiffness $)$ before peak traction is reached. One of the difficulties in the linear/softening model is to determine a criterion for peak traction which duly takes mode mixity into account. For that purpose we use a simple quadratic criterion:

$$
\left(\frac{T_{n}}{T_{n}{ }^{0}}\right)^{2}+\left(\frac{T_{t}}{T_{t}^{0}}\right)^{2}=1
$$

where $T_{n}$ and $T_{t}$ are the normal and tangential components of the traction $\bar{T}, T_{n}^{0}$ is the maximum traction in mode $\mathrm{I}$ and $T_{t}^{0}$ is the maximum traction in mode II. Thus, for any combination of traction components $\left(T_{n}, T_{t}\right)$, peak values $\left(T_{n}{ }^{i}, T_{t}{ }^{i}\right)$ are reached when the quadratic criterion is satisfied. Once peak traction is attained, softening sets in with linearly decreasing traction. The separation energy per unit area (i.e. the work of the traction) $G_{c}(\psi)$, depends on the mode mixity $\psi$. This relation is the main component of the model. It is actually independent from the peak traction criterion. Following [13], we use a phenomenological expression for the interface toughness $G_{c}$ :

$$
G_{c}(\psi)=G_{I c}\left(1+\tan ^{2}(\eta \psi)\right)
$$

with $G_{I c}$ the mode I separation energy (or toughness), and a parameter $\eta$ used to tune the mode II dependence. Values of $\eta$ close to zero imply a weak dependence on the mode mixity, while values close to unity imply a strong dependence.

Note that the measure of the mode mixity $\psi$ in our cohesive law is defined in terms of the combination of tractions attained at the peak, i.e. $\tan (\psi)=T_{t}^{i} / T_{n}^{i}$, for each cohesive element meeting the initiation criterion. This definition differs from that employed in linear fracture mechanics in that it is defined pointwise on the interface in the vicinity of crack front, whereas the conventional definition of mode mixity, $\psi$, is defined in terms of the modes I and II stress intensity factors at the crack front as $\tan (\psi)=K_{I I} / K_{I}$ (e.g. [13]). In both descriptions, a mode I situation corresponds to high bending and low stretching at the edge of the plate, whereas a mode II situation is one with high stretching and low bending. We have previously shown that there is good agreement between the present numerical model (especially the definition of mode mixity angle) and the standard results for plates ([11], Supplementary Material).

The numerical implementation of the model is carried out in the framework of the finite element method using the FEM code ABAQUS [14].

For the experiments, we monitored buckles generated by molybdenum coatings of thickness ranging between $50 \mathrm{~nm}$ and $200 \mathrm{~nm}$, deposited by magnetron sputtering on $300 \mu \mathrm{m}$ thick silicon wafers with a native oxide layer. A thin Ag layer (about $10 \mathrm{~nm}$ thick) is deposited onto the substrate prior to Mo deposition 
in order to ensure a relatively low and well controlled adhesion. Delamination occurs at the $\mathrm{Ag} / \mathrm{SiOx}$ interface.

\section{Initial destabilization of a circular blister}

\subsection{Growth of a circular defect}

Consider a film submitted to an equibiaxial compressive stress $\sigma_{0}$. A circular blister of initial radius $R_{0}$ will expand as long as the normalized driving force for delamination $G / G_{c}(\psi)$ is larger than 1 . The mode mixity dependence of the interface toughness, given here by Eq. 2, plays a key role in the existence of an equilibrium radius. The energy release rate $G$ increases monotonically with increasing radius, but so does $G_{c}(\psi)$ because $|\psi|$ increases with radius as well. As a result of this competition, the energy release rate $G$ needed to advance the interface crack increases as the blister spreads. Crack arrest is obtained if interface toughness increases sharply enough with mode $I I$.

Actually, circular growth may eventually become unstable as the radius increases as shown in [13] where the sensitivity of the circular crack front to harmonic perturbation and subsequent destabilization are studied using linear stability analysis. It is shown that the loading ratio, defined as the ratio of the residual stress to the critical buckling stress $\sigma_{0} / \sigma_{c}$, selects the number of lobes $n$ formed along the circular front. The formation of lobes is associated with a non-uniform distribution of crack driving force along the crack front and a modulation of mode mixity. Such modifications of the crack front enable further growth of the lobes.

\subsection{Incipient destabilization of the circular blister}

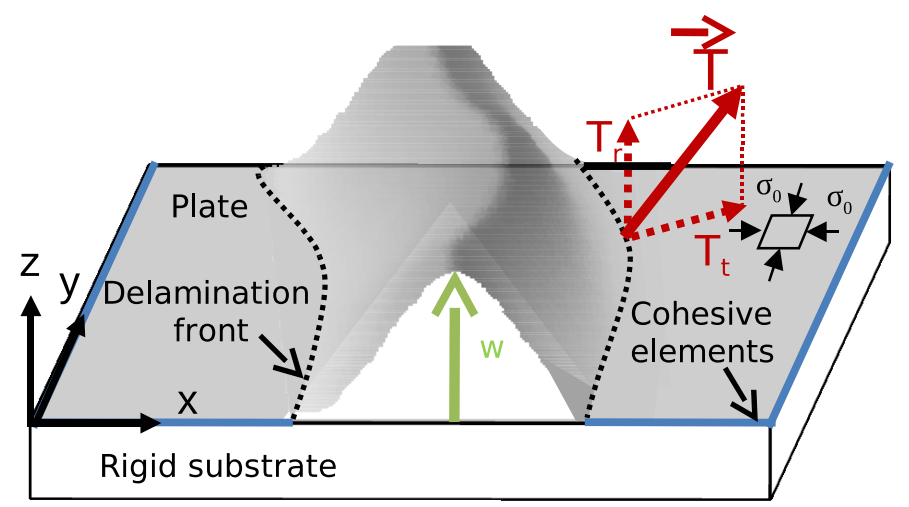

Figure 2: Model for thin film buckling and delamination: a nonlinear plate on a rigid substrate with a mixed mode cohesive zone model for the adhesive interface. $w$ indicated the out-ofplane deflection of the film due to buckling. 
We denote

$$
G_{0}=\frac{1-\nu}{E} h \sigma_{0}^{2}
$$

the elastic strain energy per unit surface stored in the film. $E$ is Young's modulus, $\nu$ Poisson's ratio and $h$ the film thickness. Given a set of adhesion parameters $\left(G_{I c}, \eta\right)$ (Eq. 2), there is a maximum $\psi$ that can be reached at the delamination front during debonding, $\psi_{\mathrm{lim}}$. We then have $G_{c}=G_{0}$ or:

$$
\psi_{\lim }=\frac{1}{\eta} \arctan \sqrt{\left(\frac{G_{0}}{G_{I c}}-1\right)}
$$

In our simulations, a value of $\eta=0.95$ is usually taken, and $\psi_{\text {lim }}$ ranges between $36^{\circ}$ and $82^{\circ}$ depending on the ratio $G_{0} / G_{I c}$.

An initial debonded zone is set as a circular defect of radius $R_{0}$. Upon compressive loading, the initial defect buckles into a circular blister when $\sigma_{0}=$ $\sigma_{c}$. Here the critical buckling stress $\sigma_{c}$ is given by:

$$
\sigma_{c}=1.2235 \frac{E}{1-\nu^{2}}\left(\frac{h}{R_{0}}\right)^{2}
$$

An example of evolution of the blister is shown in Fig. 3, where the out-ofplane displacement is plotted at various growth stages.
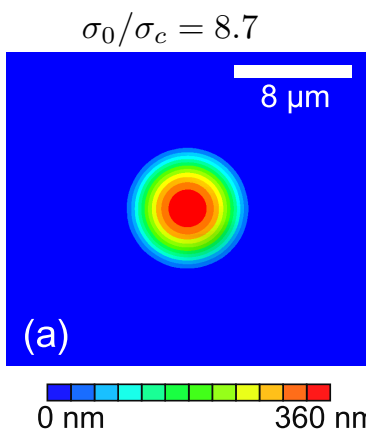

(a)

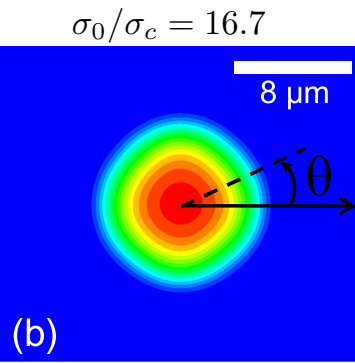

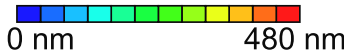

(b)
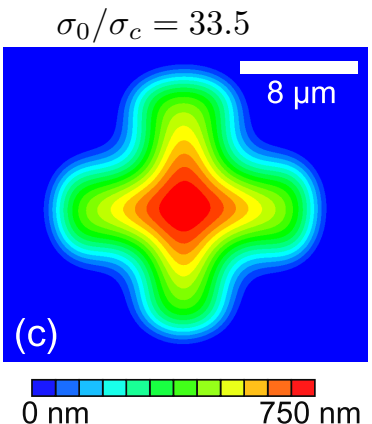

(c)

Figure 3: Various stages of growth of a circular defect submitted to an increasing $\sigma / \sigma_{c}$ loading ratio:(a) Circular growth. (b) Maximum radius before destabilization. (c) Post-destabilization morphology of the circular defect with four lobes. The polar angle $\theta$ used for the description of the mode mixity along the crack front is also reported.

In the first stage of growth, the blister remains circular (Fig. 3-b). For higher values of the loading ratio, destabilization of the circular front is observed, and the blister evolves into a non-axisymmetric morphology (Fig. 3-c). In the given example we find four lobes, with a convex local geometry, separated by concave areas where the film has sagged.

In order to better understand this phenomenon, we plot the local mode mixity along the crack front (Fig. 4) as a function of the polar angle $\theta$ (see Fig. 


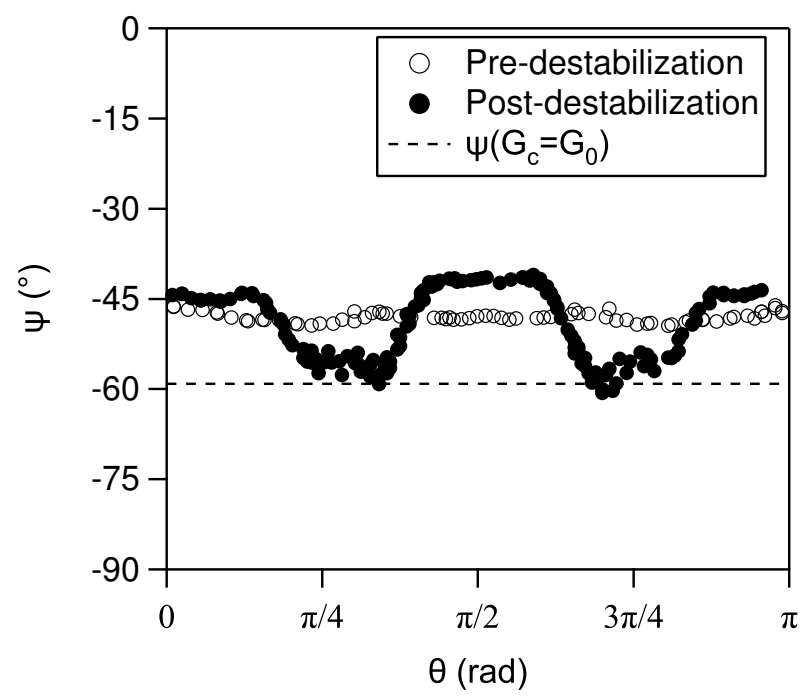

Figure 4: Local mode mixity $\psi$ along the crack front of an initially circular blister, as a function of the polar angle $\theta$ (see Fig. 3). Two states are reported: a circular shape before stabilisation and a 4 lobes shape after destabilization.

3). In the non-axisymmetric stage, we find alternating areas with lower and higher values of mode mixity. The mode mixity at the crack front is close to $\psi_{\text {lim }}$ in the concave (collapsed) regions, where the tensile contribution (mode II) is maximum; it is close to $|\psi| \approx 40^{\circ}$ in the convex (lobe) regions where mode $\mathrm{I}$ is enhanced. As interfacial toughness is mode dependent, a decrease in mode mixity promotes crack growth whereas high mode mixity keeps the front pinned. Note that, at this early stage of destabilization, there are four identical lobes, in agreement with the $n$ lobe perturbation used in [13].

As previously stated, the loading ratio $\sigma_{0} / \sigma_{c}$ is the critical parameter for destabilization of the circular buckle and directly affects the number of lobes upon destabilization. In Fig. 5 we plot the number of lobes obtained as a function of $\sigma_{0} / \sigma_{c}$ for various $G_{0} / G_{I c}$ and $\sigma_{0}$. The analytical results of [13] are also reported. We observe that the number of lobes formed upon destabilization follows the trend found analytically $([13])$ and increases gradually with $\sigma_{0} / \sigma_{c}$. However, we observe that with a cohesive zone, the same number of lobes $n$ is formed at a larger loading ratio, especially for small $n$ values. We believe this deviation is due to a finite size effect of the crack process zone. The process zone stabilizes the edge of the buckle as the stress is less released over its width. In the numerical simulations, the process zone width $\Lambda$ is about $6 h$ for the range of interface properties (i.e. peak traction components and mode I toughness) chosen. For large loading ratios, or large radii, the ratio $\Lambda / R$ diminishes and the effect of the process zone vanishes as seen in Fig. 5 .

The loading ratio is a convenient parameter to characterize the destabilization of circular buckles. However it is computed from the radius of the circular 


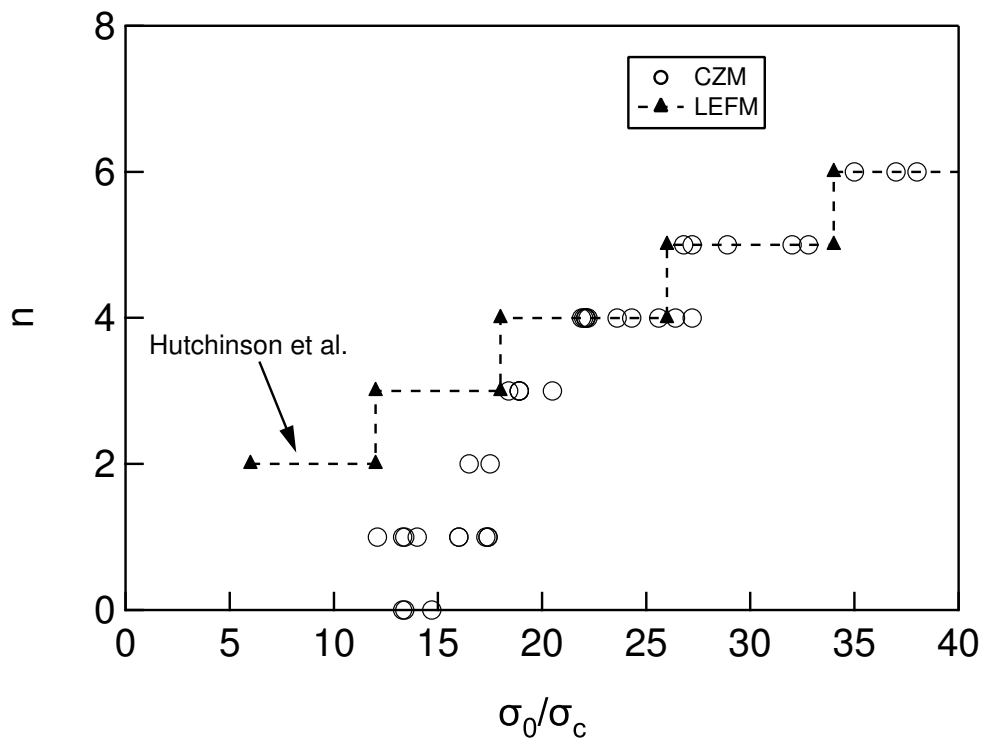

Figure 5: Number of lobes $n$ resulting from the unstable growth of an initially circular blister. Results from numerical simulation with a cohesive zone are reported for $\eta=0.95$ and $\nu_{f}=0.31$ (labelled CZM) and from [13] (labelled LEFM) for $\eta=1$ with $\alpha=0$ and $\nu_{f}=1 / 3$

buckle (i.e. initial defect) so that it is not an intrinsic parameter of the problem. Therefore, it cannot provide a criterion for growth of TC and branching buckles. We will propose an alternative critical parameter in section 5 .

\section{Branching in the propagation regime}

In the previous section, our investigations have been restricted to the study of the very first stage of the destabilization process, where the $n$ lobes are equivalent, following $[13,15]$. In order to understand the subsequent evolution of the blister morphologies out of this initial unstable defect, we have to explore the conditions under which lobes further propagate or come to a halt, eventually leading to the experimentally observed morphologies (Fig. 1). This involves computation of $G$ along a rather complex crack front, which is handled by numerical calculations.

\subsection{Case of the single $T C$ blister}

The TC morphology is characterized by a width $2 b$ and a wavelength $\lambda$ (Fig. 6 ). It was shown in a previous study [11] that during propagation of the buckle the crack front is pinned in the sagged areas due to the mixed-mode dependence of interfacial toughness. To investigate the evolution of the mode mixity along the buckle edge during propagation and branching, we plot $\psi$ along the crack front (Fig. 6) between two collapsed points $A$ and $B$ taken on each side of the 

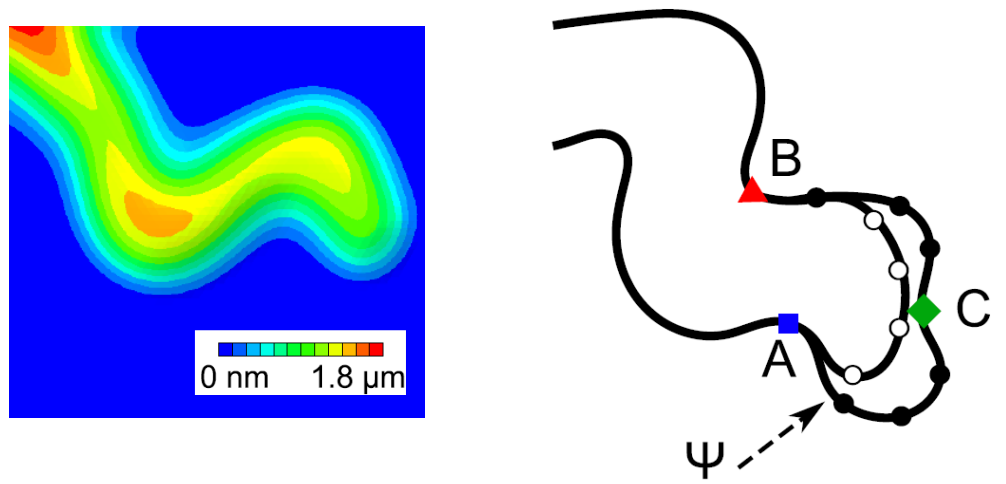

Figure 6: Out-of-plane displacement during propagation of a telephone cord buckle. Three points are introduced to support discussion about the mode mixity evolution along the crack front. The front originally developing between the two pinning points $A$ and $B$ progressively extends. A new pining point $C$ eventually forms $\left(G_{I c} / G_{0}=0.34\right.$ and $\left.\sigma_{0} / E^{*}=6.1 \times 10^{-3}\right)$.

TC and forming half a wavelength of a TC buckle. Point $B$ is located behind $A$ along the propagation trail of the TC. As the crack front moves forward during

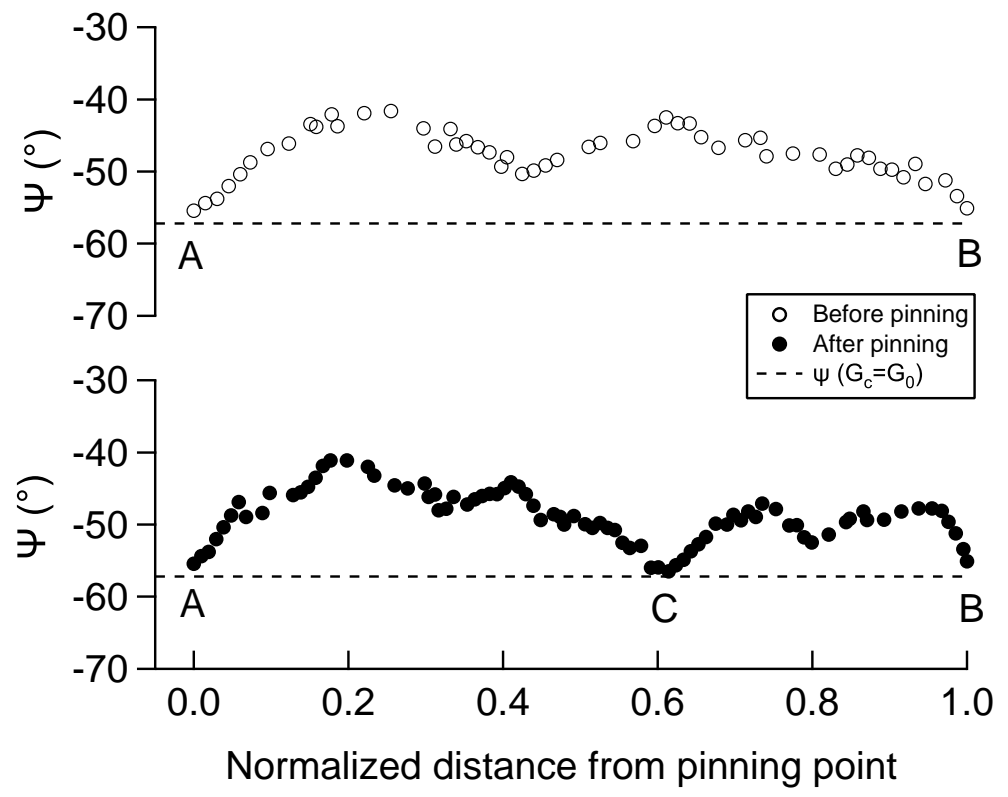

Figure 7: Evolution of the mode mixity parameter $\psi$ along the propagating front settling between the two pinning points $A$ and $B$ (see Fig. 6). The crack front length between $A$ and $B$ is normalized. Two states are depicted: before and after the development of a new pinning point $C$. 
propagation, the distance $A B$ is normalized to 1 . The first curve corresponds to the state of the crack front after formation of point $A$. We observe the high mode mixity, $|\psi| \approx \psi_{l i m}$ in the sagged areas around $A$ and $B$. Between $A$ and $B$ however, $|\psi|$ is lower. Note that the mode mixity is not constant in these areas, unlike the case of the circular blister destabilization for which a plateau is observed. This observation suggests that the propagating front of the TC buckle is not quite equivalent to a circular blister. After propagating further, we observe the formation of another area with $|\psi| \approx \psi_{\text {lim }}$ around point $C$, which becomes a new pinning point. Interestingly, point $C$ does not appear at middistance between $A$ and $B$. The position of $C$ varies during propagation but is definitely closer to $B$. In addition, the average mode mixity over $A C$ is lower (in absolute value) than between $B C$. We believe this difference is the source of arrest of the front $B C$ whereas front $A C$ will keep propagating and form a new undulation. We have found a similar kinematics for all the TC buckles we have simulated.

A critical value for the formation of telephone cord buckles seems to be correlated to the value of the loading ratio $\sigma_{0} / \sigma_{c}$ upon destabilization of the circular buckle. In fact, no TC buckle was observed for values below $\sigma_{0} / \sigma_{c} \approx 16$ nor above $\sigma_{0} / \sigma_{c} \approx 22$, a rather limited range.

\subsection{Case of branching blisters}

Above $\sigma_{0} / \sigma_{c} \approx 22$, the growth of lobes still occurs but in this case the morphology obtained, while still periodic, develops a branching structure. Separation of the crack front into two branches is visible on both experimental propagation and numerical simulations on Fig. 8. It is interesting to note that the transition between isolated TC buckles and branching structures is not sharp, as it is possible to simultaneously observe the two structures under the same conditions, both numerically and experimentally (Fig. 1). To better understand branching, we plot $\psi$ along the propagating crack front of a buckle (Fig. 9) for the three different states depicted in Fig. 8. Locations of points $A, B, C$ and $D$ are indicated in Fig. 8. The distance between $A B$ is normalized to 1 . In the first frame, captured after the formation of pinning point $A$, the mode mixity is rather constant, except in the neighbourhood of pinning points $A$ and $B$, and similar to the plateau state observed for the lobes upon destabilization of a circular buckle (Fig.4). After propagation, we observe the formation of pinning point $C$ between $A$ and $B$ similar to the TC case. However the position of point $C$ differs as it is right at mid-distance between $A$ and $B$. The consequence of the position is that the average $|\psi|$ on both sides of $C$ is lower than in the TC case. As a result none of the crack fronts arrest: they both propagate. Later on, the symmetry between the two propagating branches is broken as another pinning point $D$ appears between $C$ and $B$. The consequence is a lower $|\psi|$ along $C D$ than $D B$. This difference causes arrest of the $D B$ front while both $A C$ and $C D$ fronts keep propagating. 

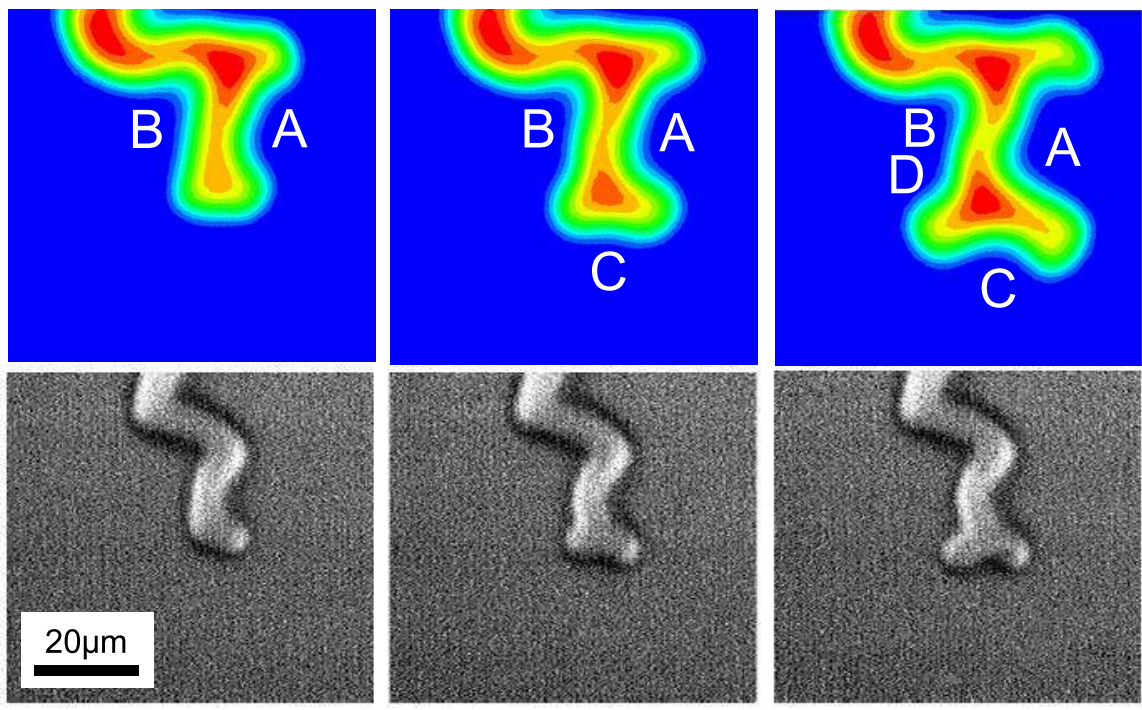

Figure 8: Detailled snapshot of the out of plane displacement of a blister during branching at the front both numerically (top) and experimentally (bottom). In the experiment, the fracture occurs at the $\mathrm{SiOx} / \mathrm{Ag}$ interface, with $15 \mathrm{~nm}$ of $\mathrm{Ag}$ loaded by $132 \mathrm{~nm}$ of $\mathrm{MoOx}$ $\left(\sigma_{0}=1.84 G P a\right)$. For the simulation, the parameters are chosen so that $G_{I c} / G_{0}=0.34$ and $\sigma_{0} / E^{*}=6.1 \times 10^{-3}$. Pinning points are introduced in the top picture to support discussion about the evolution of mode mixity along the crack front during propagation.

\section{A phase diagram for thin film blisters}

The loading ratio impacts both the wavelength of the TC buckle and the ability of the front to experience branching. This combination of phenomena gives rise to the wide variety of morphologies observed experimentally. Starting from a rectangular defect, and by varying the parameters of the model, we have simulated a variety of buckling morphologies shown in Fig. 10 under the form of a phase diagram. Each point indicates a calculated morphology for different h, $\sigma_{0}$ and $G_{I c}$.

We observe that two simple reduced parameters provide an adequate description of this phase diagram. The fist parameter is $G_{I c} / G_{0}$ which is the ratio of the interface toughness to the total elastic energy stored by unit surface in the film. The second parameter is directly the normalized residual stress $\sigma_{0} / E^{*}$, with $E^{*}=E /(1-\nu)$. With these two parameters, we find a clear separation between three regions (Fig. 10). For large values of mode I interfacial toughness $G_{I c}$, the initial defect is stable: no propagation is observed. By decreasing $G_{I c}$ while keeping the stress $\sigma_{0}$ constant, we first find the TC blister followed by branching blister and complete delamination when interfacial toughness decreases further.

In this diagram, the boundary between the 'no delamination' and the 'telephone cord' areas can be approximated by a straight line. The condition for 


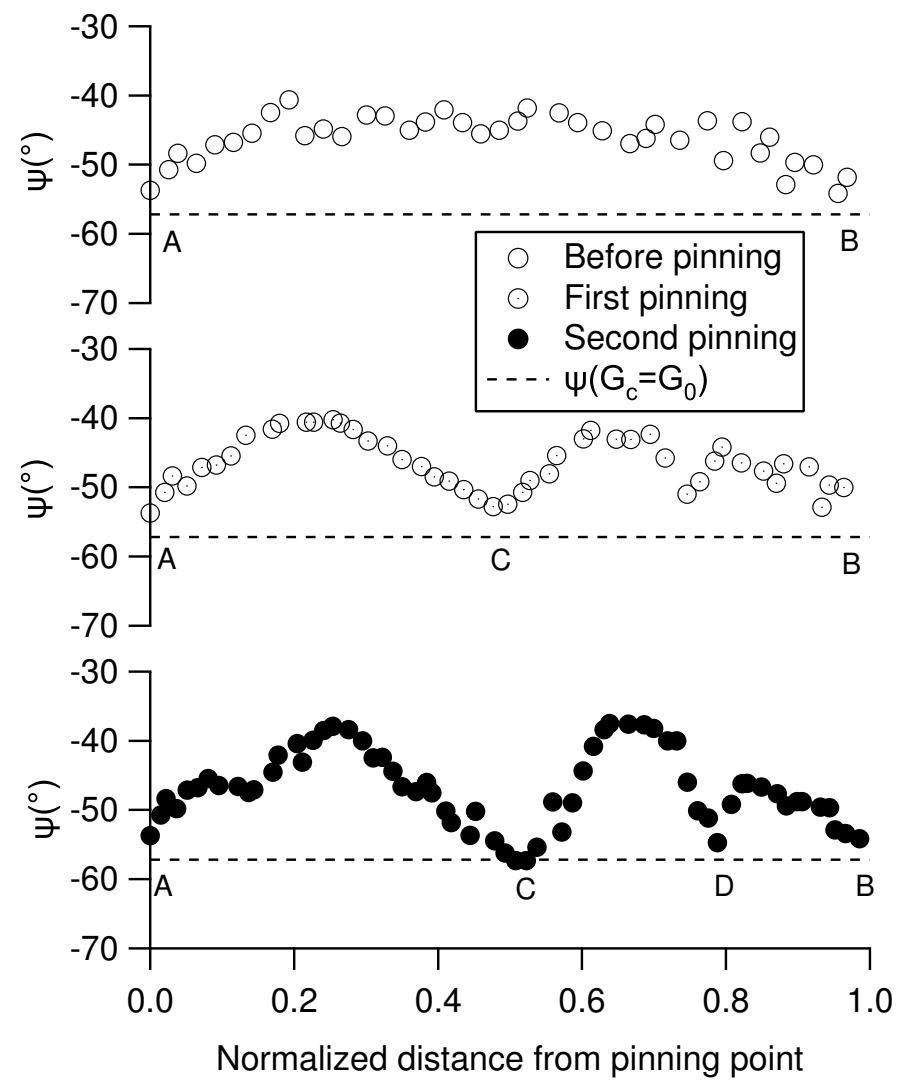

Figure 9: Evolution of the mode mixity parameter $\psi$ along the propagating front settling between the two pinning points $A$ and $B$ (see Fig. 8). The crack front length between $A$ and $B$ is normalized. Three states are depicted: a uniform plateau of $\psi$ between $A$ and $B$, the development of a third pinning point $C$ in the middle and finally the development of a fourth pinning point $D$ breaking the previous symmetry.

buckle delamination to occur can be expressed as:

$$
\frac{\sigma_{0}}{E^{*}} \geq c \frac{G_{I c}}{G_{0}}
$$

where $c$ is a positive constant.

The limit between TC buckles and branched structures is slightly more difficult to define. Note that the boundary may move a little depending on the mesh size adopted in the simulation. Here the element sizes were scaled with $h$ and $\bar{E} / \sigma_{0}$ so that the number of elements along $R$ for an equivalent circular blister would remain constant. Also, two boundaries may be considered: 1) first appearance of a branch in the morphology or 2) complete disappearance of the TC morphology. One possible boundary is plotted in Fig. 10. We find that this boundary is also linear in the present parameter space. 


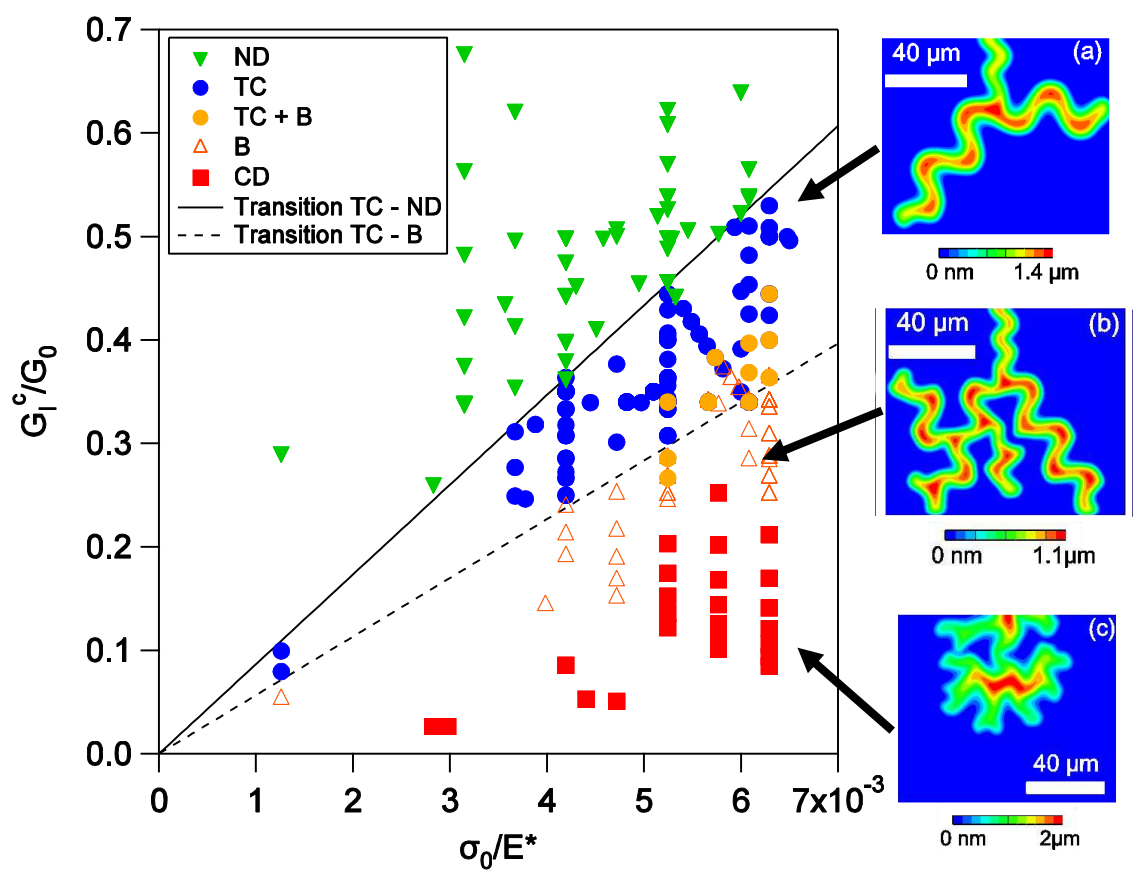

Figure 10: Phase diagram of the delamination morphology with $\eta=0.95$ and $\nu=0.31$. The morphology of delamination is indicated (symbols) and the calculated limit for propagation from an initial (solid line) defect and branching of a delamination front (dashed line) are figured. ND : No delamination, TC : Telephone cord, B : Branching, CD : Complete delamination.

The value $\eta=0.95$ that has been chosen here characterizes an interface toughness that is highly sensitive to mode mixity. In this regime, the choice of $\eta$ is not expected to qualitatively influence the phase diagram. To evaluate quantitative modifications, the two cases $\eta=0.95$ and $\eta=0.8$ are plotted in Fig. 11. The linear boundaries are scaled up by roughly the same factor (about 1.25) when $\eta$ decreases from 0.95 to 0.8 .

\section{Discussion}

In view of the previous results, inequality 6 can be rewritten as $\sigma_{0} \geq \sigma_{c}^{*}$ with

$$
\sigma_{c}^{*}=c_{1}\left(E^{*}\right)^{2 / 3}\left(\frac{G_{I c}}{h}\right)^{1 / 3}
$$

which defines a new critical stress $\sigma_{c}^{*}$. For $\eta=0.95$ and $\nu=0.31, c_{1} \approx 0.23$. It is interesting that, unlike the critical buckling stress $\sigma_{c}$ (Eq. 5), this new critical stress is intrinsic since it does not require the introduction of an extrinsic reference length (e.g. radius of a defect). 


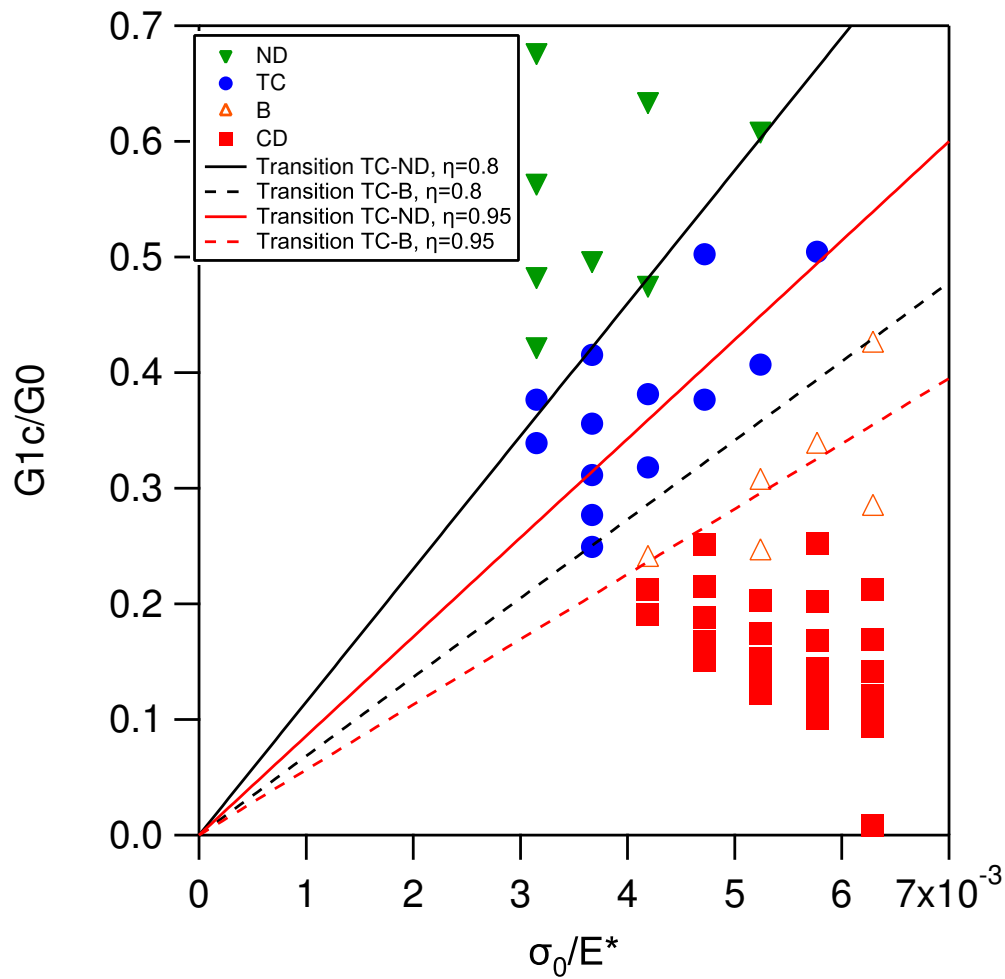

Figure 11: Phase diagram of the delamination morphology with $\eta=0.8$. The lines defining the boundaries between the stability regions have been inserted. The boundary lines obtained in the case $\eta=0.95$ have been recalled.

In fact, Eqs. 5 and 7 define a characteristic size $L^{*}$ with

$$
\frac{L^{*}}{h}=\sqrt{\frac{E^{*}}{\sigma_{c}^{*}}} \simeq\left(\frac{E^{*} h}{G_{I c}}\right)^{1 / 6}
$$

Rough estimates suggest that in our systems, $L^{*}$ is of the order of 10 times the film thickness. The prefactor varies weakly with the model parameters. This intrinsic lengthscale is quite different from the telephone cord wavelength we have defined previously [12]. Here a natural interpretation is that for defect sizes larger than $L^{*}$, buckles will propagate while smaller defects will remain stable. It does not mean that the initial defect can be taken arbitrarily though. If the initial defect is larger than the resulting blister size, the initial mode mixity will be too large for propagation $\left(G_{c}>G_{0}\right)$. But provided that the initial defect is taken small compared to the resulting buckle size after propagation, its size does not influence the final result.

We observe that for $\sigma_{0} / \sigma_{c}^{*}=1.07$ the first branching events appear in the phase diagram. TC blister are still observed up to $\sigma_{0} / \sigma_{c}^{*}=1.15$, above which 
almost only branching blisters are observed. We point out that for identical thickness and interfacial toughness, a small variation of stress may lead to a strong variation of the observed morphology. Such variations are common in the Mo thin films presented above.

Comparing the positions of the phase boundaries for the two values of $\eta$ we have investigated, we observe that for a given value of $G_{I c} / G_{0}, \sigma_{c}^{*}$ seems to decrease with $\eta$. In other words, smaller values of $\eta$ promote destabilization. This observation is consistent with earlier results [13] which show that harmonic destabilization occurs at lower compression for smaller $\eta$.

Interestingly, we did not observe any propagation of straight sided blister in the numerical simulations for equi-biaxial loaded films with the mode II dependence chosen $(\eta=0.95)$. This result is in accordance with Jensen [16] who predicted almost immediate instability of a straight front for such loading. We expect the straight sided morphology to appear for smaller vales of $\eta$ for low values of $G_{I} c / G_{0}$ and $\sigma_{0} / E^{*}$. It seems from the diagram that the domain of stability of the TC buckle is narrow. However, experimentally, TC buckles are observed over a wide range of thicknesses and stress levels. This is not contradictory with the results of or analysis, but is actually related to the way stresses build up in thin films during deposition. For example if we assume that the stress is kept constant and variation of $G_{0}$ arises solely from the thickness, TC buckles may be observed over a range of thicknesses. If $h_{0}$ is the thickness of the film at the onset of propagation, TC buckle will be observed up to $1.5 h_{0}$.

To illustrate this effect, in Fig 12 we plot the evolution of $G_{I c} / G_{0}$ as a function of $\sigma_{0} / E^{*}$ for two experimental molybdenum thin films taken from a previous study [17]. The superlayer is deposited on top of an interface with an interfacial toughness in mode I of $G_{I c}=0.4 \mathrm{~J} . \mathrm{m}^{-2}$ and it is assumed that no relaxation occurs in the thin film after deposition.

- For $\mathrm{MoO}_{\mathrm{x}}$ thin film, the range over which stable TC buckles are predicted spans from $195 \mathrm{~nm}$ to $283 \mathrm{~nm}$, which is consistent with a rapid destabilization of the TC buckle.

- For pure Mo, TC buckles are expected from $230 \mathrm{~nm}$ up to $670 \mathrm{~nm}$. This larger range of stability is due to a strong decrease of the average residual stress through the thickness and a small increase in $G_{0}$, which is common in Mo thin films past the first nanometers.

Figure 12 also shows that in the case of the Mo film, given the evolution of the stress and the thickness during the deposition process, it is actually possible to enter and exit the telephone cord buckles stability area to end up inside the 'no delamination' area. It is hence possible to get thick unbuckled films. Indeed, it is usually observed that the buckles develop once the sample have been taken out of the deposition chamber ; so the final state reached by the sample is likely to be the most critical. These observations can be related to the nucleation process of buckle delamination, which is still poorly understood. 


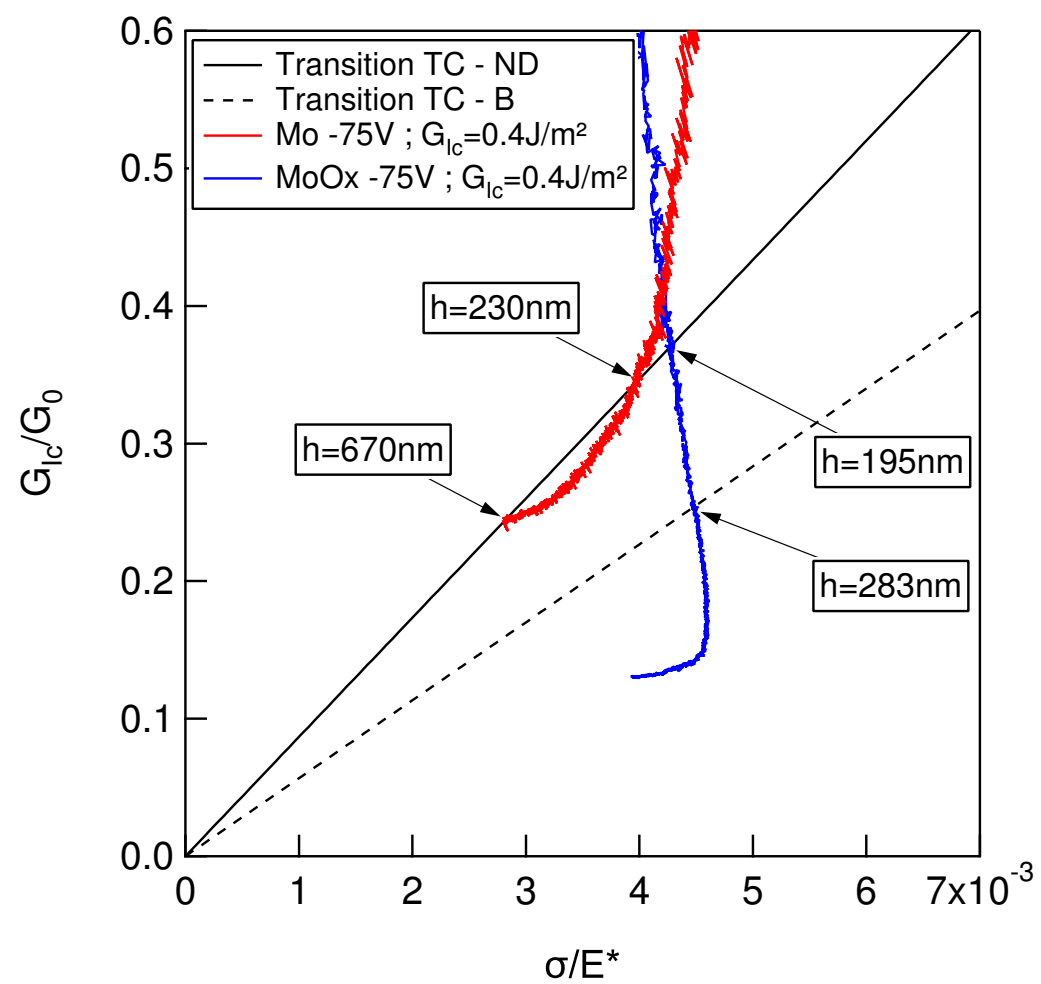

Figure 12: Possible energy range available with two different superlayer, Mo and $\mathrm{MoO}_{\mathrm{x}}$ at $-75 \mathrm{~V}$ assuming an interface with $G_{I c}=0.4 \mathrm{~J} \cdot \mathrm{m}^{-2}$. The experimental data are superimposed to the phase diagram (Fig. 10). The thicknesses at the crossing of the boundaries between the stability domains are displayed.

\section{Conclusions}

A model coupling non-linear plate buckling and cohesive zone has been used to study buckling driven delamination under equi-biaxial compressive stress and mode-dependent interfacial toughness. The results obtained numerically are well correlated with experimental observations. The critical role of plate instability resulting in interface pinning is identified not only for initial circular defect, but also latter on in the process of buckle propagation for both undulating and branching mechanisms of the crack front.

Both undulating and branching mechanisms are very similar and differ only by the different position of the pining point $C$ when the instability develops. In the case of the standard TC, the locus of the point $C$ breaks the symmetry and leads to selection between an arrested branch and a propagating one. In the case of branching, the pinning point $\mathrm{C}$ appears right in the middle of the two candidate branches, which both propagate further. Those two mechanisms are responsible for the generation of the patterns observed experimentally, in 
particular the telephone-cord like blister.

It has been possible to obtain a phase diagram of the buckling driven delamination morphology based on those two mechanisms. Two boundaries have been identified from the phase diagram: propagation of a crack front from an initial defect and branching of the crack front. With the help of the diagram, we have been able to define a critical stress that is intrinsic to the system, i.e. only depending on the film elasticity modulus, thickness and stresses as well as adhesion. This critical stress also defines an intrinsic size $L^{*}$ which may play the role of a minimum size for propagation. The boundaries on the diagram, which can be determined from the intrinsic data of the system, are of critical importance, whether to avoid propagation of buckles on experimental samples or to control the buckling induced patterning in some way (branching or not). A practical use of the analysis results has been illustrated in the specific case of $\mathrm{Mo}$ and $\mathrm{MoO}_{\mathrm{x}}$ films.

[1] L.D. Landau, E.M. Lifchitz, Theory of Elasticity, 3rd ed., Pergamon, New York, 1986.

[2] Hutchinson J. W., Suo Z., Mixed Mode Cracking in Layered Materials, Adv. Appl. Mech. 29 (1992) 63-191.

[3] G. Gille, B. Rau, Buckling instability and adhesion of carbon layers, Thin Solid Films 120 (1984) 109-121.

[4] J.S. Wang, A.G. Evans, Effects of strain cycling on buckling, cracking and spalling of a thermally grown alumina on a nickel-based bond coat, Acta Materialia 47 (1999) 699-710.

[5] S.-J. Yu, M.-G. Chen, J. Chen, H. Zhou, Y.-J. Zhang, P.-Z. Si, Spatial and kinetic evolutions of telephone cord buckles, Surf. Coat. Technol. 228 (2013) 258-265.

[6] G. Parry, A. Cimetière, C. Coupeau, J. Colin, J.Grilhé, Stability diagram of unilateral buckling patterns of strip-delaminated films, Phys. Rev. E 74 (2006) 066601.

[7] B. Audoly, Stability of straight delamination blisters, Phys. Rev. Lett. 83 (1999) 4124.

[8] Audoly B., Roman B., Pocheau A., Secondary buckling patterns of a thin plate under in-plane compression, J. Eur. Phys. J. B 27 (2002) 7-10.

[9] M. W. Moon, H. M. Jensen, J. W. Hutchinson, K. H. Oh et al., The characterization of telephone cord buckling of compressed thin films on substrates, J. Mech. Phys. Solids 50 (2002) 2355-2377.

[10] Moon M.-W., Lee K.-R., Oh K. H., Hutchinson. J. W., Buckle delamination on patterned substrates, Acta Mater. 52 (2004) 3151-3159. 
[11] Faou J.-Y., Parry G., Grachev S., Barthel E., How does adhesion induce the formation of telephone cord buckles?, Phys. Rev. Let. 108 (2012) 116102.

[12] J.-Y. Faou, G. Parry, S. Grachev, E. Barthel, Telephone cord buckles: A relation between wavelength and adhesion, J. Mech. Phys. Solids 75 (2015) 93-103.

[13] Hutchinson J. W., Thouless M. D., Liniger E. G., Growth and configurational stability of circular, buckling-driven film delaminations, Acta metall. mater. 40 (1992) 295-308.

[14] ABAQUS Manuals Collection, Dassault Systèmes Simulia Corp., Providence, RI, USA, 2010.

[15] Nilsson K.-.F., Giannakopoulos A. E., A finite element analysis of configurational stability and finite growth of buckling driven delamination, J. Mech. Phys. Solids 43 (1995) 1983-2021.

[16] H.M. Jensen, M.D Thouless, Effects of residual stresses in the blister test, Int. J. Solids Structures 30(6) (1993) 779-795.

[17] Faou J.-Y., Barthel E., Grachev S. Y.,Stress tuning in sputter-deposited MoOx films, Thin Solid Films 527 (2013) 222. 